\title{
MODELLING OF HYDRODYNAMICS AROUND AN IMPERMEABLE BREAKWATER: COMPARISON BETWEEN PHYSICAL AND SPH NUMERICAL MODELING
}

\author{
E. Didier ${ }^{\mathrm{a}, \mathrm{b}}$, \\ D. R. C. B. Neves ${ }^{a}$, \\ R. Martins ${ }^{\mathrm{a}, \mathrm{b}}$, \\ and M. G. Neves ${ }^{\mathrm{a}}$ \\ ${ }^{a}$ Laboratório Nacional de Engenharia Civi \\ Núcleo de Portos e Estruturas Maritimas \\ Av. do Brasil, 101, 1700-066, \\ Lisboa, Portugal \\ edidier@lnec.pt \\ dneves@lnec.pt \\ gneves@lnec.pt \\ ${ }^{\mathrm{b}}$ Universidade Nova de Lisboa, \\ Faculdade de Ciências e Tecnologia \\ 2829-516, Monte de Caparica, \\ Portugal \\ deric@fct.unl.pt
}

Keywords: SPH, Breakwater, Wave Breaking, Wave Overtopping, Pressure

This work presents the new developments and the validation of a Smoothed Particle Hydrodynamics (SPH) numerical model used in the National Laboratory of Civil Engineering (Laboratório Nacional de Engenharia Civil - LNEC) for studies in coastal engineering processes. Although the model requires a high CPU time, it proved to be very promising in the simulation of complex flows, such as the wave-structure interaction and the wave breaking phenomenon. For the SPH model validation, physical modeling tests were performed in one LNEC's flume to study the interaction between an impermeable structure and an incident regular wave. The comparison between numerical and experimental results, i.e. free surface elevation, overtopping volume and pressure, shows the good accuracy of the SPH model to reproduce the various phenomena involving on the wave propagation and interaction with the structure, namely the wave breaking, the wave overtopping and the pressure field on the structure.

\section{NOMENCLATURE}

$c_{0} \quad$ Sound velocity, $\mathrm{m} / \mathrm{s}$

$d \quad$ Depth, m

$d_{0} \quad$ Particle spacing, $\mathrm{m}$

$f \quad$ Function

$g \quad$ Gravity acceleration, $\mathrm{m} / \mathrm{s}^{2}$

$h \quad$ Smoothing length, $0.92 \times 2^{0.5} d_{o}$, m

$H \quad$ Wave height, $\mathrm{m}$

$L \quad$ Wavelength, m

LNEC Laboratório Nacional de Engenharia Civil

$m \quad$ Mass, kg

$P \quad$ Pressure, $\mathrm{Pa}$

Ps Pressure $\mathrm{mH}_{2} \mathrm{O}, \mathrm{m}$

$q \quad$ Non-dimensional distance between particles

$r \quad$ Particle position (vector), $\mathrm{m}$

$t \quad$ Time, $\mathrm{s}$

SPH Smoothed Particle Hydrodynamics

$\mathrm{T} \quad$ Wave Period, $\mathrm{s}$

$v \quad$ Velocity (vector), $\mathrm{m} / \mathrm{s}$

$V_{r} \quad$ Overtopping volume $\left(\mathrm{m}^{3} / \mathrm{m}\right)$

$W \quad$ Weighting function (kernel)

$W_{a b} \quad$ Value of kernel at position $\left(r_{a}-r_{b}\right)$

$\tilde{W}_{a b} \quad$ Corrected kernel

$x, z \quad$ Cartesian coordinates, $\mathrm{m}$

$Z_{r} \quad$ Wave run-up, $\mathrm{m}$

\section{Greek symbols}

$\rho \quad$ Density, $\mathrm{kg} / \mathrm{m}^{3}$

\author{
$\rho_{0} \quad$ Reference density of water, $1000 \mathrm{~kg} / \mathrm{m}^{3}$ \\ $\Pi \quad$ Viscous terms \\ $\eta \quad$ Free surface elevation, $\mathrm{m}$ \\ $\nabla_{a} W_{a b}$ Kernel gradient at position $\left(r_{a}-r_{b}\right)$
}

\section{Subscripts \\ a Particle a \\ $b \quad$ Particle b}

\section{INTRODUCTION}

Over the last decade the numerical modeling in fluid dynamics became a relevant tool due to the recent developments on the computational methods. The equations describing the flow are known for a long time, but with the improvement and the development of the computational techniques it has become easier to obtain approximate solutions to these equations and consequently to quickly simulate realistic scenarios. The great advantages of the numerical modeling relate to the fact that it represents a faster and a less expensive method comparing with the physical modeling, also the flexibility enabling an easy change in the geometry or on the wave conditions by simply changing the input data of the model. Due to these characteristics the numerical modeling is increasingly used to complement the physical modeling, allowing acquiring information 
about the flow where it would be very difficult or even impossible to obtain in the physical model.

For wave-structure interaction studies, that include wave breaking, wave overtopping and wave impact forces on maritime structures, there are currently several numerical models, each one with its advantages and limitations. Among the existing numerical models, one can highlight the three different types of models that are currently in development or/and validation at LNEC: AMAZON model (Hu, 2000), based on the nonlinear shallow water equations; IH-2VOF model (Lara et al., 2011), based on the RANS equations (Reynolds-AveragedNavier-Stokes), and the SPH model (Monaghan, 1994) based on a Lagrangian method with the concept of Smoothed Particle Hydrodynamics (SPH). The models AMAZON and IH-2VOF have already been successfully applied for wave-structure interaction studies: the first model is applied especially for the study of the overtopping of impermeable structures and it is being developed/validated for porous structures and the second model presents good results regarding studies of wave interaction with porous structures. The SPH model is under development and validation.

The SPH numerical model developed at LNEC is based on the original SPHysics model, version 1.4 (http://wiki.manchester.ac.uk/sphysics, 2009), inspired by the original Monaghan (1994) formulation. In this model, a weakly compressible fluid technique is used, i.e. pressure is calculated by an equation of state and function of the fluid density. The SPH model has been developed and validated at LNEC for wave-structure interaction applications, specifically for wave breaking, wave overtopping, wave reflection and forces on maritime structures (Didier and Neves, 2008, 2009a, 2009b, 2009c, 2010a, 2010b, 2012a, 2012b; Didier et al., 2011; Neves et al., 2012). The development of the numerical model is based in the Lagrangian form of the fluid dynamics equations that are written in SPH formalism using the interpolation integrals theory and interpolation kernels. The SPH method is a mesh-free technique and does not require any specific formulation for the free surface, allowing the modeling of the fluid particle trajectories according to the Navier-Stokes equations and the velocity and pressure fields. Thus, SPH method presents great potential in the simulation of complex flows such as those occurring in the wave-structure interaction, wave breaking process and for the wave overtopping, although the model requires a high runtime.

In order to validate the SPH numerical model, physical modeling tests were performed regarding the interaction of incident regular waves with an impermeable breakwater with a 2:3 slope. The physical modeling tests were set with the purpose of ensuring a consistent reproduction of the same model characteristics in the numerical model: flume dimensions and piston-type wave-maker movement are the same for both numerical and physical model. Validation of the numerical model is achieved comparing time series of free surface elevation measured in four resistive wave gauges, of pressure measured at two pressure sensors placed on the breakwater slope and of overtopping volume measured in a tank located behind the structure. The tests are part of a larger set of experimental tests with the specific purpose of validating the SPH model for several structure configurations. So that, the SPH model was previously validated for regular incident waves interacting with an impermeable vertical breakwater, showing that the model can correctly estimate the free surface elevation and the force on the vertical wall of the breakwater, even for the very complex case where wave impacts the structure (Didier et al., 2011, 2012a, Martins, 2012).

Fundamental principle of the SPH methods and the numerical SPH model are firstly presented. After that, the case study and the physical modeling tests are described. In the next section, numerical results are compared with physical experiments. Finally, conclusions and an approach on the future developments of the numerical SPH model are presented.

\section{SPH NUMERICAL MODEL}

The SPH numerical methods were initially applied to the astrophysics (Gingold e Monaghan, 1977; Lucy, 1977) and were then applied to the hydrodynamics (Monaghan, 1994).

The SPH method is a completely mesh-free technique, enabling the modeling of the fluid particles trajectories accordingly to the Navier-Stokes equations written on the SPH formalism, based on the theory of interpolation integrals which use interpolation kernels. The Lagrangian approach of the SPH methods, that consists in following the fluid particles in a determined time interval in order to obtain its trajectories, velocities and pressures as a function of the initial position and time, is different from the Eulerian approach where the model requires a mesh and calculates the velocity and pressure at the mesh nodes as a function of time.

\section{Fundamental principle of the SPH method}

The fundamental principle of the SPH methods is to approximate a scalar, a function or a tensor using the theory of the interpolation integrals. The integral of an interpolation function $f(r)$ is given by:

$$
f(r)=\int f\left(r^{\prime}\right) W\left(r-r^{\prime}, h\right) d r^{\prime}
$$

where $r$ is the vector particle position, $W$ is the weighting function, called kernel, $h$ is the smoothing length. The kernel allows determining the interaction among neighboring particles included in their influence domain, a compact support within a circular 
region determined by a radius of $2 h$, controlled by the smoothing length $h$, typically higher than the initial particle spacing, $d_{0}$.

Numerically, the function $f(r)$ is only known at discrete points, the particles, and the interpolation integrals are approximated by the sum of the contribution of each particle included in their influence domain. The function is written as an approximation of the function $f$ at a particle $a$ at the position $r_{a}$, and is given by:

$$
f\left(r_{a}\right) \approx \sum_{b} m_{b} \frac{f_{b}}{\rho_{b}} W_{a b}
$$

where $f_{b}$ is the value of the function $f$ associated with the particle $b$ at $r_{b}, W_{a b}=W\left(r_{a}-r_{b}, h\right)$ is the value of the kernel at $\left(r_{a}-r_{b}\right), m_{b}$ and $\rho_{b}$ are the mass and the density of the particle $b$, respectively.

The kernel is an analytic function that can be differentiated without requiring any spatial mesh. Numerically, the kernel is a function with compact support within a region determined by a radius of $2 h$ (Figure 1), smaller than the typical scale of the problem. Meanwhile, $h$ must be, as referred before, greater than the initial particles separation, $d_{o}$. Thus, one particle should be only in interaction with the particles that are on its influence area, previously defined by the kernel dimension, and each one of these particles must have a contribution for the kernel (Figure 1).

There are several kernels in the literature (Liu, 2003). For numerical simulation of wave propagation, the quadratic kernel (Johnson et al., 1996; Dalrymple and Rogers, 2006) is used to determine the interaction between the particles. This kernel has the particularity of not having an inflection point in its first and second derivative in the range of the function definition. This kernel is defined by the analytic function:

$$
W(q, h)=\frac{3}{2 \pi h^{2}}\left(\frac{q^{2}}{4}-q+1\right) \text { for } 0 \leq q \leq 2
$$

where $q=\left(r_{a}-r_{b}\right) / h$.

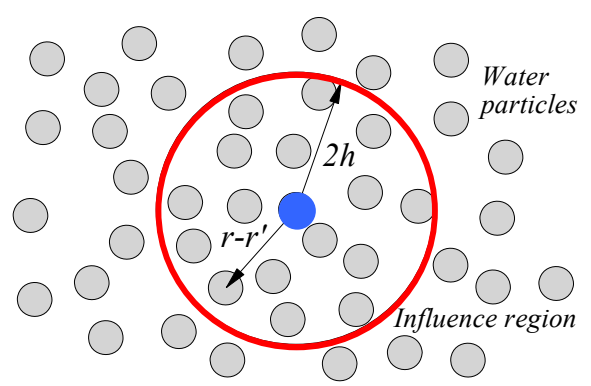

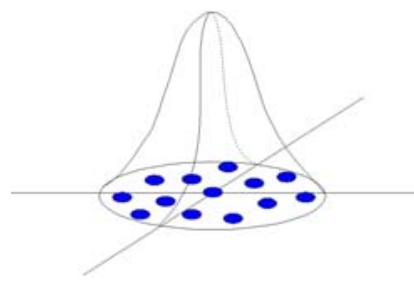

Figure 1. Influence region of the kernel, particles that contribute for the interpolation in the compact support of the kernel.

\section{Equations of Fluid Dynamics in the Lagrangian} form

The two-dimensional SPH equations are based on the Lagrangian formulation of the conservation of momentum and continuity:

$$
\begin{aligned}
& \frac{d v}{d t}=-\frac{1}{\rho} \nabla P+\Pi+g \\
& \frac{1}{\rho} \frac{d \rho}{d t}=-\operatorname{div}(v)
\end{aligned}
$$

where $t$ is the time, $\Pi$ represents the viscous terms, $g=(0,-9.81) \mathrm{ms}^{-2}$ is the acceleration of gravity, $v, P$ and $\rho$ are, respectively, the velocity, pressure and density.

The standard SPH formulation (Monaghan, 1994), in which the fluid is considered slightly compressible, is used and the pressure is calculated by an equation of state (Batchelor, 1974) through the fluid density:

$$
P=B\left[\left(\frac{\rho}{\rho_{0}}\right)^{\gamma}-1\right] \text { with } B=\frac{c_{0}^{2} \rho_{0}}{\gamma}
$$

with $\gamma=7, \rho_{0}$ the reference density and $c_{0}$ the sound velocity.

The particle trajectories are obtained from the following relationship:

$$
\frac{d r}{d t}=v
$$

where $r$ is the particle position.

\section{SPH numerical model}

The SPH numerical model used and developed at LNEC is based on the open-source code SPHysics, version 1.4 (Crespo, 2008; http://wiki.manchester. ac.uk/sphysics, 2009), inspired by the SPH standard formulation of Monaghan (1994). The fluid in the standard SPH formalism is treated as slightly compressible, allowing the direct pressure calculation through a state equation (Batchelor, 1974), that relates the fluid pressure with the density. The LNEC's SPH numerical model is specifically 
developed for solving coastal engineering problems and for modeling complex free surface flows and wave interacting with coastal structures (impermeable and porous structures).

In the SPH equations, the discrete equation of conservation of momentum is given by

$$
\frac{D v_{a}}{D t}=\sum_{b} m_{b}\left(\frac{P_{a}}{\rho_{a}^{2}}+\frac{P_{b}}{\rho_{b}^{2}}+\Pi_{a b}\right) \cdot \nabla_{a} W_{a b}+g
$$

where $t$ is the time, $g=(0,-9.81) \mathrm{m} . \mathrm{s}^{-2}$ is the gravity acceleration, $v_{a}, P_{a}$ and $\rho_{a}$ are the velocity, the pressure and the density of a particle $a$, respectively, $P_{b}, \rho_{b}$ and $m_{b}$ are the pressure, the density and the mass of a particle $b$, respectively, included in the influence region of the kernel, $W_{a b}$ is the kernel and $\Pi_{\mathrm{ab}}$ the viscosity term. Finally, $\nabla_{a} W_{a b}$ is the kernel gradient.

The turbulence model SPS - Sub-Particle Scale (Gotoh et al., 2001) is used since it includes not only a viscosity model but also the turbulence effect through a model derived from the LES-type models (Large Eddy Simulation). The artificial viscosity model (Monaghan, 1992), with two empirical parameters that should be calibrated, is frequently used to stabilize the numerical process, introducing a strong numerical diffusion that might affect significantly the wave amplitude (Didier and Neves, 2009b).

The equation of mass conservation in the discrete SPH formalism is given by:

$$
\frac{D \rho_{a}}{D t}=\sum_{b} m_{b}\left(u_{a}-u_{b}\right) \cdot \nabla_{a} W_{a b}
$$

The particles move according to the following equation:

$$
\frac{D r_{a}}{D t}=u_{a}
$$

In the LNEC's SPH model, XSPH correction term due to Monaghan (1994) is not used in equation Eq. (10). The XSPH correction is a modification for the particle velocity, which is recalculated taking into account the velocity of that particle and the average velocity of neighboring particles. However, it was shown in Didier and Neves (2009b, 2010a) that instabilities appear during the wave propagation, particles cross the solid boundary and fluid flow exhibits unphysical behaviors. by:

The equation of state (Batchelor, 1974) is given

$$
P=B\left[\left(\frac{\rho}{\rho_{0}}\right)^{\gamma}-1\right]
$$

For numerical reasons, the sound velocity $c_{0}$ must be high enough to reduce fluctuations due to density and low enough to allow the model to run with a reasonable calculation time step. Therefore, it is usually considered, for the calculations, a sound velocity significantly lower than its classic value.

The flow kinematics of the SPH models is generally realistic. However, a problem inherent in the weakly compressible SPH formulation, based on the formulation of Monaghan (1994), is the pressure of particles which can exhibit large oscillations. Gómez-Gesteira et al. (2010) shows that filtering density (i.e. renormalization of particle density) allows to obtain a pressure field without oscillations and a good representation of a dam-break flow. However, filtering density influence seems to be more complex for wave propagation. Hughes et al. (2010) compared ISPH (Incompressible SPH solver) and WCSPH (Weakly Compressible SPH solver) models, with and without filtering density, for waves impacting on a vertical wall. Both methods have shown to be able of simulating the pressure on the vertical wall due to the wave impacts. The predictions for the wave impacts have shown to be in agreement, qualitative and quantitative, with the available experimental data. Results from the WCSPH appear to be significantly smoother than those from ISPH. This is particularly the case for the free surface deformation and especially for the wavebreaking. Preliminary studies (Didier et al., 2011, 2012a; Martins, 2012) comparing numerical and experimental results for wave impact on a vertical breakwater showed that the conventionally used density filter stabilizes the pressure field but causes a numerical diffusion on wave propagation and a consequent reduction on wave height that is not observed in the experimental results. On the other hand, without the density filter, pressure oscillations occur and forces on structures are more difficult to be calculated. With these results, a partial filtering density technique, i.e. renormalization applied only for particles near the structure, is developed and allows simultaneously propagating waves, without diffusion, and modeling accurately the pressure field near the structure, without oscillations.

The pressure instabilities can be resolved by correcting the kernel function, recalculating the value of the particle density. The application of the density renormalization is performed every 30 iterations of time (classical value) applying the Shepard filter (Panizzo, 2004). The new density value of a given particle is calculated by:

$$
\begin{aligned}
& \bar{\rho}_{a}=\sum_{b} m_{b} \tilde{W}_{a b} \\
& \tilde{W}_{a b}=\frac{W_{a b}}{\sum_{b} W_{a b} \frac{m_{b}}{\rho_{b}}}
\end{aligned}
$$


In the LNEC's SPH model, the time integration is performed using the Predictor-Corrector algorithm (Monaghan, 1989). The time step is automatically controlled accordingly to the conditions proposed by Monaghan and Kos (1999). The particle velocity is calculated using the discrete equation of the momentum conservation Eq. (8), the density is set by the discrete form of the continuity equation Eq. (9) and position of particles is calculated by Eq. (10). The pressure is calculated from the density using the Tait equation (11).

\section{Boundary conditions and initial conditions}

The boundary conditions are not displayed directly in the SPH formalism. In the present model the repulsive boundary condition, developed by Monaghan and Kos (1999), that imposes a repulsive force from the solid particles of the boundary to the fluid particles, is used and allows preventing the water particles to cross the solid boundary.

Initially, the water particles are placed in the flume using a regular Cartesian distribution, i.e. particles are regularly distributed, with the spacing between particles defined by $d_{o}$. This is a condition of the SPH method when the smoothing length of the kernel is constant. The distribution of the solid particles at boundaries follows the one adopted for the fluid particles, namely the distance between the particles is equal to $d_{o}$ independently of the direction of the boundaries.

Initially, the velocity field is zero and the pressure is hydrostatic. The particle density is equal to the water density, $1000 \mathrm{~kg} / \mathrm{m}^{3}$. Figure 2 presents a view of the initial distribution of solid and fluid particles near the vertical breakwater and the correspondent hydrostatic pressure.

\section{Active wave-maker absorption for a semi-infinite wave flume}

In order to simulate a semi-infinite numerical wave flume, a piston-type wave-maker active absorption, instead of simple wave-maker, was implemented in the SPH numerical model (Didier and Neves, 2012b). Active wave-maker absorption follows the same procedure as in physical flumes: the numerical wavemaker is equipped with a control system for

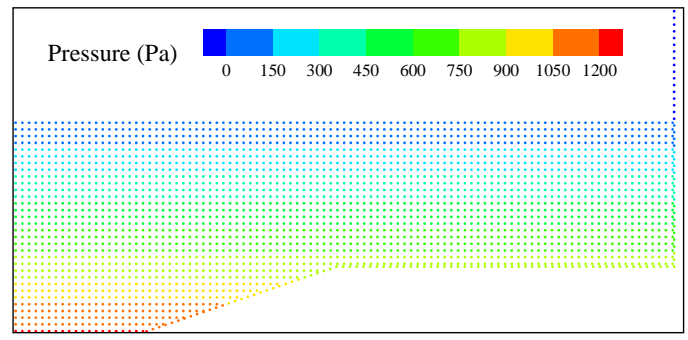

Figure 2. Typical initial distribution of solid and fluid particles and correspondent hydrostatic pressure. simultaneous wave generation and active wave absorption of the reflected waves. The model enables to obtain longer time series of free surface elevation, overtopping and forces, needs for a correct calculation of the statistical parameters, without undesired influence of re-reflection of waves at the wave-maker.

A recent modification in the numerical model for the active wave-maker absorption technique was the correction of the paddle drift from its initial position over time, observed for some simulation settings. It is a phenomenon that also occurs in the experimental wave channel and must be corrected. The principle for the drift correction of the paddle in the numerical wave channel is similar to one used in the experimental wave flume, specifically the correction of the paddle drift is performed in real time in order to maintain the average position of the wave-maker close from its initial position (Neves et al., 2012).

\section{PHYSICAL MODELLING}

The physical modeling tests here presented were performed in one irregular wave flume (COI1) at LNEC. The flume has a total length of $49.40 \mathrm{~m}$, with a $1.60 \mathrm{~m}$ width $(0.80 \mathrm{~m}$ useful wide) and a height of $1.20 \mathrm{~m}$. The wave generation is done with a piston type wave-maker with an active absorption system for the reflected waves (AWASYS - Active Wave Absorption System). However, for the tests here presented the AWASYS system was not used.

The tested geometry represents an impermeable breakwater with a sloping wall of 2:3. The breakwater built in acrylics is based on a hood structure with a 1:3 slope ramp. The structure is placed on a horizontal bottom and at $3.62 \mathrm{~m}$ from the wave-maker. The characteristics of the physical model have been defined in order to make it feasible to the numerical model. The dimensions of flume and of the breakwater are defined in Figure 3.

For the conducted tests four resistive-type wave gauges were used. The wave gauges were placed, respectively, in a distance of $2.64 \mathrm{~m}$ (gauge G1), $3.36 \mathrm{~m}$ (gauge G2), $3.62 \mathrm{~m}$ (gauge G3) and $3.94 \mathrm{~m}$ (gauge G4) from the wavemaker. Two pressure sensors, P1 and P2, were placed in the slope of the structure to acquire the pressure. The center of the pressure sensor $\mathrm{P} 1$ is placed at the first third of the slope, from the beginning of the structure $(4.262 \mathrm{~m}$, $0.2543 \mathrm{~m}$ ) and the second pressure sensor P2 is at second third $(4.372 \mathrm{~m}, 0.3276 \mathrm{~m})$. Overtopping volume, $V_{r}$, is measured by the variation of the water depth in a tank located behind the structure, where the water that overtops the structure was collected. The experimental data was acquired with a frequency of $400 \mathrm{~Hz}$ (Didier et al., 2011, 2012a; Martins, 2012).

The experimented wave conditions were a combination of wave heights, $H$, from $0.08 \mathrm{~m}$ to $0.14 \mathrm{~m}$, periods, $T$, from $1.1 \mathrm{~s}$ to $1.6 \mathrm{~s}$ and flume depths 
near the wave-maker, $d$, equal to $0.266 \mathrm{~m}$ and $0.325 \mathrm{~m}$. A total of 12 waves were simulated in order to have a useful simulation time to compare with the SPH numerical model. Each wave condition was repeated ten times. Test repetitions present a good agreement.

These are complex experimental tests, where the set of the involved physical processes change significantly the waveform characteristics along its propagation, due to wave breaking and its reflection on the structure, wave overtopping and the wave reflection at the wave-maker.

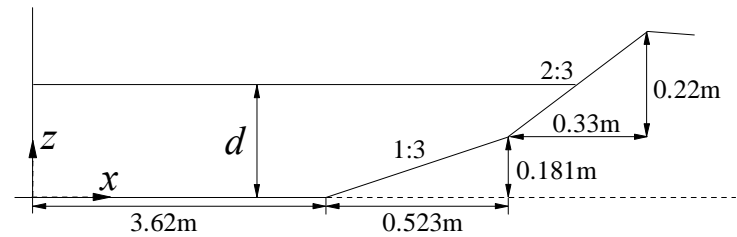

Figure 3. Schematic representation of the impermeable breakwater and flume.

\section{COMPARISON OF EXPERIMENTAL AND NUMERICAL RESULTS}

The computational domain, as well as the physical domain is, on the left side, constituted by the piston-type wave-maker without active absorption, and on the right by the studied coastal structure. The adopted dimensions for the numerical model are the same as the physical model.

For the model validation, one regular incident wave was chosen, with $1.3 \mathrm{~s}$ period and $0.14 \mathrm{~m}$ height, with a depth of the flume of $0.325 \mathrm{~m}$. The regular wave generation, as referred, is made with a pistontype wave-maker with the same amplitude as the experimental tests, corresponding to $0.7076 \mathrm{~m}$ for the wave height of $0.14 \mathrm{~m}$.

The SPS viscosity model, the quadratic kernel and the Predictor -Corrector time integration scheme are used. The re-normalization of the density was not used in the present case (2010a).

The adopted resolution of the computational domain for the simulation is shown in Table 1 and is defined based on the experience gain of using $\mathrm{SPH}$ for wave propagation and overtopping of impermeable coastal structures. Particle dimension, $d_{o}$, is $2.321 \times 10^{-3} \mathrm{~m}$ which corresponds to 247259 particles. Resolution for flume depth, $d$, wave height $H$, and the wavelength $L$ is also indicated.

Table 1. Resolution and number of particles.

\begin{tabular}{cccccc}
\hline \multirow{2}{*}{$\boldsymbol{H}(\mathbf{m})$} & \multirow{2}{*}{$\boldsymbol{d}_{\boldsymbol{o}}(\mathbf{m})$} & \multirow{2}{*}{$\mathbf{N}^{\mathbf{2}}$ part. } & \multicolumn{3}{c}{ Resolution } \\
& & & $\boldsymbol{H}$ & $\boldsymbol{L}$ \\
\hline 0.14 & 0.002321 & 247259 & 140 & 60 & 870 \\
\hline
\end{tabular}

The numerical simulation time is $12.0 \mathrm{~s}$, such as the experimental tests and the average time step is around $1.7 \times 10^{-5} \mathrm{~s}$.
The CPU time for each wave period is approximately 22 hours in a computer PC Intel Core(TM) i7 930 with $2.80 \mathrm{GHz}$ which corresponds to 204 hours for simulating 12.0s of flow.

The numerical results of the free surface elevation in the four gauges (G1 to G4), the pressure in the two sensors (P1 e P2) and the overtopping volume $(V r)$ are compared with the experimental data. The wave run-up $\left(Z_{r}\right)$ is also presented, although run-up was not measured in the physical tests.

Figures 4 to 7 show: (i) the time series of free surface elevation in the 4 gauges, G1 to G4; (ii) the overtopping volume; (iii) the pressure at P1 and P2 sensors and (iv) the run-up obtained with the numerical SPH model.

Free surface, Figure 4, is well simulated by the numerical model, presenting a good agreement with the experimental data, indicating that the used resolution, either horizontally or vertically, is well adapted to the problem of the wave propagation and of the interaction between the incident and the reflected wave by either the structure or the wavemaker. However, there are major differences after 8 to 9s in gauge G4, located in the middle of the 1:3 slope, due to effects of the re-reflected waves from the wave-maker that reach the gauge. Despite the strong non-linear effects of the re-reflection of the wave-maker, the free surface elevation in the gauges G1 to G3 is well simulated by the model, presenting a very good agreement with the physical model measurements, where the same effects are observed.

The overtopping volume, Figure 5, obtained with the numerical model presents a very good agreement with the experimental data. From results obtained with other conditions (wave height, wave period and water depth), it seems that the number of particles per wave height is a critical parameter for the resolution definition and to ensure accuracy of the overtopping modeling, which is the more complex phenomena to model, since is strongly dependent from all the others processes. It seems necessary to consider at least 60 particles per wave height in order to obtain numerical results that are independent from the resolution, which is the case of the presented simulation. A more detailed analysis of the wave overtopping volume per wave is showed later in Table 2.

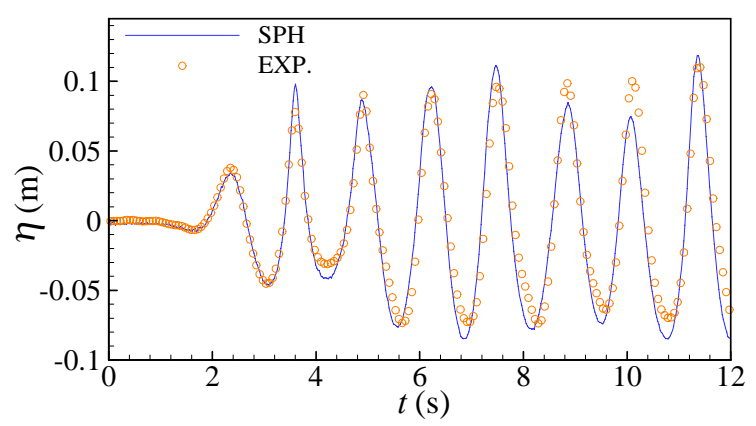



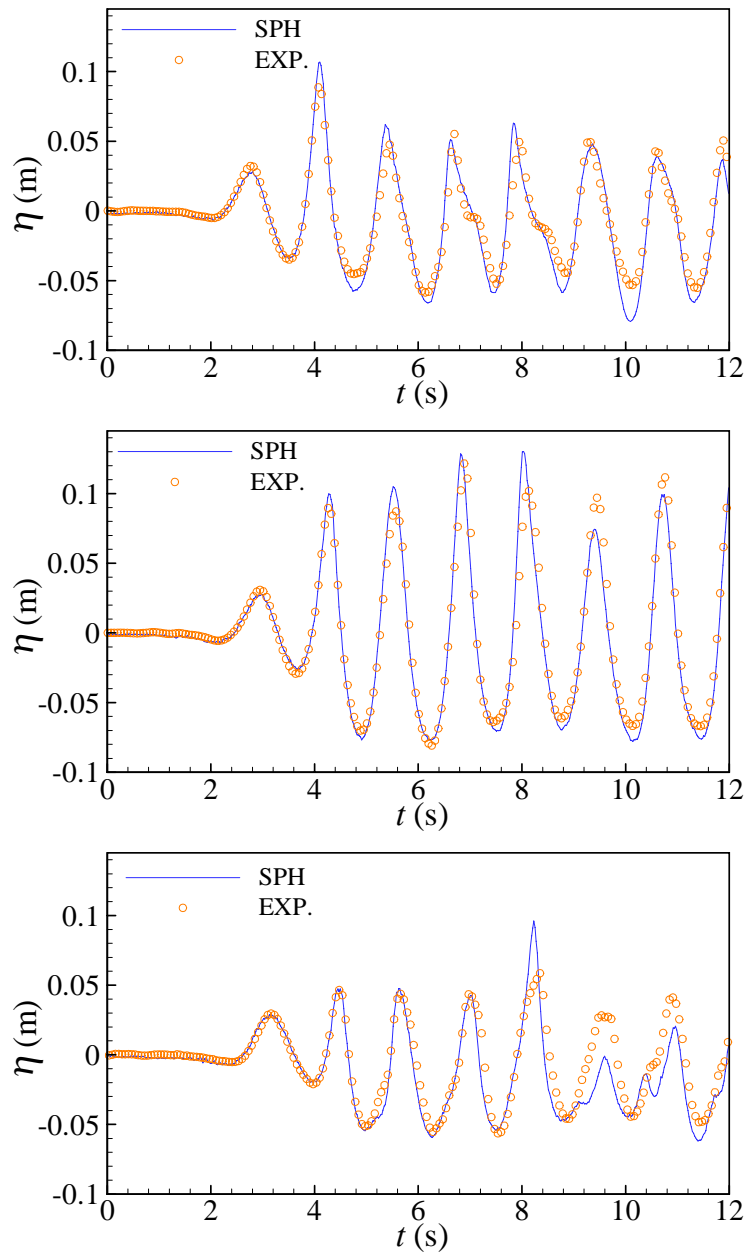

Figure 4. Free surface elevation obtained with the SPH numerical model and the physical model at the gauges G1, G2, G3 and G4.

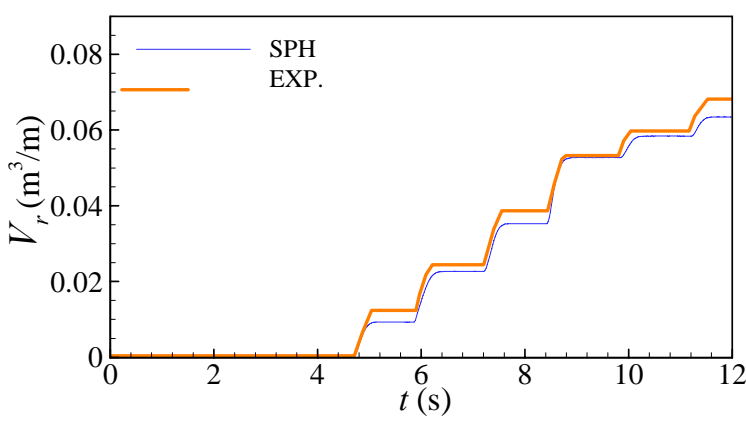

Figure 5. Overtopping volume for the SPH numerical model and the physical model.

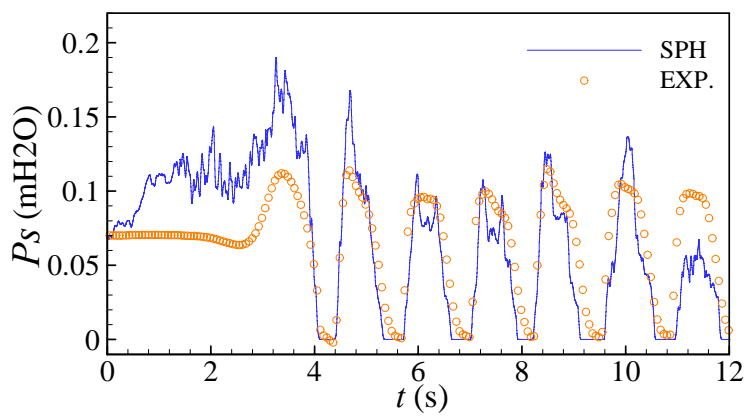

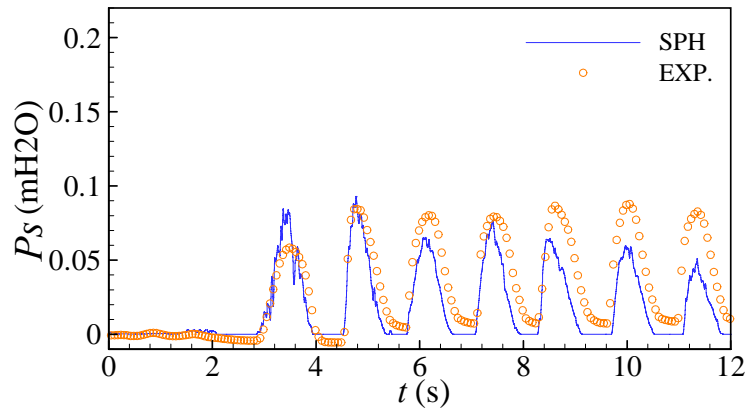

Figure 6. Pressure obtained with the SPH numerical model and with the physical model in P1 and P2 pressure sensors.

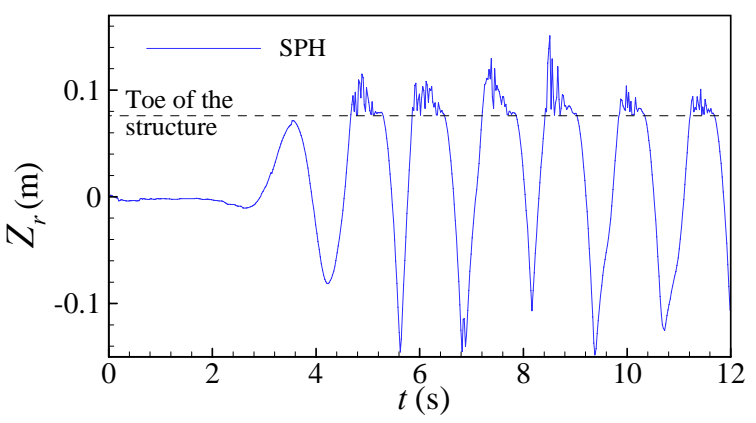

Figure 7. Run-up obtained with the SPH numerical model.

The pressure variation in the two pressure sensors, Figure 6, is globally well estimated. The most significant differences are observed after 9-10s, when the waves re-reflected by the wave-maker interact with the breakwater. The agreement between the numerical and the experimental results in the pressure sensor $\mathrm{P} 1$ is better than in the pressure sensor P2. This can be endorsed to inherent limitations of the pressure sensors when the wave periods are short (such as the used testing period, $T=$ 1.3s) and the sheets of water over the sensor are small, reducing the accuracy of the measurements. As referred before, the re-normalization allows the stabilization of the pressure but induces a strong reduction in wave height and overtopping volume (Didier et al., 2011). Because of that, the density renormalization is not used in the SPH simulation, and, consequently, pressure exhibits fluctuations, particularly before the wave reaches the structure (before 4s).

The run-up is presented Figure 7. The mean greenwater height above the toe of the structure is around $0.028 \mathrm{~m}$, with a minimum of $0.018 \mathrm{~m}$ and a maximum of $0.064 \mathrm{~m}$.

The overtopping volume per meter of structure and for each wave (waves W1 to W6) obtained by physical model and by the numerical model is presented in Table 2. Comparing numerical and experimental results, it appears that the accumulated overtopping volume of the six waves has a difference of $9.7 \%$. For the overtopping volume per wave, the average difference is of $27.2 \%$. The agreement 
between the numerical results and the experimental data is very good regarding the very complex flow. The resolution seems appropriated for the problem since wave overtopping is well modeled, i.e. the model provides accurate results for wave breaking on the breakwater and for interaction between the incident wave and the flow due to the run-down.

Figure 8 shows, for two time instants, the particle positions near the breakwater. In these figures it can be seen the interaction between the incident wave and the reflected wave and the wave overtopping over the breakwater.

Table 2. Overtopping volume and overtopping volume per wave from numerical and physical model.

\begin{tabular}{|c|c|c|c|c|}
\hline & Wave & SPH & Exp. & Error \% \\
\hline \multirow{7}{*}{ 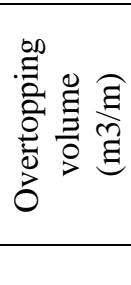 } & W1 & 0.0093 & 0.0130 & 28.44 \\
\hline & W2 & 0.0227 & 0.0238 & 4.66 \\
\hline & W3 & 0.0353 & 0.0398 & 11.25 \\
\hline & W4 & 0.0527 & 0.0534 & 1.20 \\
\hline & W5 & 0.0584 & 0.0613 & 4.76 \\
\hline & W6 & 0.0634 & 0.0688 & 7.83 \\
\hline & Wave & SPH & Exp. & Error \% \\
\hline \multirow{6}{*}{ 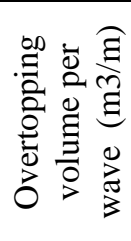 } & W1 & 0.093 & 0.0130 & 28.44 \\
\hline & W2 & 0.0134 & 0.0108 & 24.00 \\
\hline & W3 & 0.0126 & 0.0160 & 21.07 \\
\hline & W4 & 0.0174 & 0.0136 & 28.14 \\
\hline & W5 & 0.0570 & 0.0790 & 28.61 \\
\hline & W6 & 0.0050 & 0.0075 & 32.89 \\
\hline
\end{tabular}
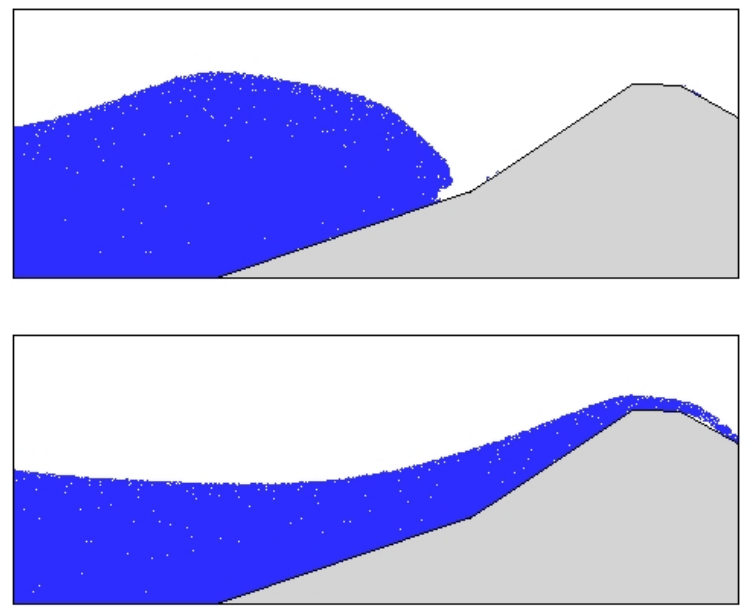

Figure 8. Image snapshot of the particles for two instants during a wave period.

\section{CONCLUSIONS AND FUTURE DEVELOPMENTS}

The SPH numerical model is being developed at LNEC to solve problems regarding the coastal engineering. The model has been validated and applied to study the wave propagation and the wave overtopping for different coastal structures.

Nevertheless, to validate and deeply analyze the performance of the SPH numerical model, physical modeling tests were carried out at LNEC regarding the interaction of regular incident waves with an impermeable breakwater. These tests were set to ensure a consistent reproduction in the numerical model: boundary conditions are equal to the physical model, i.e. wave generation is made using a pistontype wave-maker with the same amplitude motion, and the dimensions of the computational domain are equal to the physical flume. The wave interaction with the breakwater is very challenging in the computational fluid dynamics, since it includes several complex and nonlinear phenomenon, such as wave breaking and reflection, impact of the wave on the structure and the wave overtopping.

The good accuracy of the results from the numerical simulations with the measurements in the physical model tests for an impermeable breakwater showed the capability of SPH model to simulate accurately the complex issues involved in the overtopping phenomenon and to predict the average flow and the forces (pressures) over a coastal structure.

\section{ACKNOWLEDGEMENTS}

The first author acknowledges the financial support of the Portuguese foundation for the science and technology (Fundação para a Ciência e a Tecnologia - FCT) SFRH/BPD/37901/2007. The authors acknowledge the FCT for the financial support through the project PTDC/ECM/114109/2009, "SPACE - A Smoothed Particle Hydrodynamic model development and validation for coastal engineering applications”.

\section{REFERENCES}

Batchelor, G. K., 1974, Introduction to Fluid Dynamics, Cambridge University Press, UK.

Crespo, A. J. C., 2008, Application of the Smoothed Particle Hydrodynamics model SPHysics to Free-Surface Hydrodynamics, Doctoral Thesis, Universidade de Vigo, Espanha.

Dalrymple, R. A., and Rogers, B. D., 2006, Numerical Modeling of Water Waves with the SPH Method, Coastal Engineering, Vol. 53, No. 2-3, pp. 141-147.

Didier, E., and Neves, M. G., 2008, Estudo da Interacção Onda-Estrutura Utilizando um Modelo Numérico Lagrangiano, Revista Recursos Hidricos, Vol. 29, No. 2, pp. 15-26. (in Portuguese)

Didier, E., and Neves, M. G., 2009a, Wave Overtopping of a Typical Coastal Structure of the Portuguese Coast Using a SPH Model, Journal of Coastal Research, Vol. SI 56, pp. 496-500.

Didier, E., and Neves, M. G., 2009b, Coastal Flow Simulation Using SPH: Wave Overtopping on an Impermeable Coastal Structure, in: 4th International SPHERIC Workshop, Nantes, France, pp. 357-364.

Didier, E., and Neves, M. G., 2009c, Método Lagrangiano para Modelação Numérica da Interacção 
entre uma Onda e uma Estrutura Costeira, in: Jornadas Portuguesas de Engenharia Costeira e Portuária, Funchal-Madeira, Portugal. (in Portuguese)

Didier, E., and Neves, M. G., 2010a, Study of Wave Interaction with Coastal Structures Using a SPH Numerical Model, Journal of Integrated Coastal Zone Management, Vol. 10, No. 4, pp. 435-455.

Didier, E., and Neves, M. G., 2010b, A Lagrangian Smoothed Particles Hydrodynamics SPH - Method for Modelling Waves-Coastal Structure Interaction, in: CFD2010 ECCOMAS, Lisboa, Portugal.

Didier, E., Martins, R., Neves, M. G., and Vasco, J., 2011, Interaction Between Wave and Coastal Structure: Validation of two Lagrangian Numerical Models with Experimental Results, in: MARINE 2011 - Computational Methods in Marine Engineering IV, Lisbon, Portugal, pp. 134-145.

Didier, E., Martins, R., Neves, D.R.C.B., and Neves, M.G., 2012a, Modelação Física e Numérica da Interacção entre uma Onda Regular e um QuebraMar Vertical, in: IV Conferência Nacional em Mecânica dos Fluidos, Termodinâmica e Energia, Lisbon, Portugal. (in Portuguese)

Didier, E., and Neves, M. G., 2012b, A SemiInfinite Numerical Wave Flume Using Smoothed Particle Hydrodynamics, International Journal of Offshore and Polar Engineering - IJOPE, Vol. 22, No. 3, pp. 193-199.

Gingold, R. A., and Monaghan, J. J., 1977, Smoothed Particle Hydrodynamics: Theory and Application to Non-Spherical Stars, Monthly Notices of the Royal Astronomical Society, Vol. 181, pp. 375-389.

Gómez-Gesteira, M., Rogers, B. D., Dalrymple, R. A., and Crespo, A. J. C., 2010, State-of-the-Art of Classical SPH for Free-Surface Flows, Journal of Hydraulic Research, Vol. 48, Extra Issue, pp. 6-27.

Gotoh, H., Shibahara, T., and Sakai, T., 2001, Sub-Particle-Scale Turbulence Model for the MPS Method-Lagrangian Flow Model for Hydraulic Engineering, Computational Fluid Dynamics Journal, Vol. 9, No. 4, pp. 339-347.

Hu, K., Mingham, C. G., and Causon, D. M., 2000, Numerical Simulation of Wave Overtopping of Coastal Structures Using Non-Linear Shallow Water Equations, Coastal Engineering, Vol. 41, pp. 433465.

Hughes, J. P., and Graham, D. I., 2010, Comparison of Incompressible and WeaklyCompressible SPH Models for Free-Surface Water Flows, Journal of Hydraulic Research, Vol. 48, Extra Issue, pp. 105-117.

Johnson, G., Stryk, R., and Beissel, S., 1996, $\mathrm{SPH}$ for High velocity Impact Calculations, Computer Methods in: Applied Mechanics and Engineering, Vol. 139, pp. 347-373.

Lara, J. L., Ruju, A., and Losada, I. J., 2011, Reynolds Averaged Navier-Stokes Modelling of Lon
Waves Induced by Transient Wave Group on a Beach, R. Soc. A., Vol. 467, pp. 1215-1242.

Liu, G. R., 2003, Mesh Free Methods. Moving Beyond the Finite Element Method, CRC press.

Lucy, L. B., 1977, A Numerical Approach to the Testing of the Fission Hypothesis, Astronomical Journal, Vol. 82, No. 12, pp. 1013-1024.

Martins, R., 2012, Analise da Interacçao entre Uma Onda Regular e um Quebra-Mar Vertical Usando um Modelo Numérico Smoothed Particle Hydrodynamics, Master Thesis, Universidade Nova de Lisboa, Lisbon, Portugal. (in Portuguese)

Monaghan, J. J., 1989, On the Problem of Penetration in Particle Methods, Journal Computational Physics, Vol. 82, pp. 1-15.

Monaghan, J. J., 1992, Smoothed Particle Hydrodynamics, Annual Review of Astronomy and Astrophysics, Vol. 30, pp. 543-574.

Monaghan, J. J., 1994, Simulating Free Surface Flows with SPH, Journal of Computational Physics, Vol. 110, pp. 399-406.

Monaghan, J. J., and Kos, A., 1999, Solitary Waves on a Cretan Beach, Journal of Waterways, Ports, Coastal and Ocean Engineering, Vol. 125, pp. $145-154$

Neves, D. R. C. B., Didier, E., and Reis, M. T., Neves, M. G., 2012, Overtopping of a Porous Structure Using a Smoothed Particle Hydrodynamics Numerical Model, in: 4th International Conference on the Application of Physical Modelling to Port and Coastal Protection, Ghent, Belgium.

Panizzo, A., 2004, Physical and Numerical Modelling od Sub-aerial Landslide Generated Waves, Doctoral Thesis, Universita degli Studi di L'Aquila, Roma, Italia.

Received: September 30, 2012

Revised: October 30, 2012

Accepted: November 30, 2012 